More Efficient Shielding for Internal than External Attention? Evidence from Asymmetrical

9

\section{Switch Costs}

Sam Verschooren ${ }^{1}$, Gilles Pourtois ${ }^{1}$, Tobias Egner ${ }^{2}$

${ }^{1}$ Ghent University, Ghent, Belgium; ${ }^{2}$ Duke University, North Carolina, USA

Word count: 9.955

Corresponding author

Sam Verschooren

Cognitive \& Affective Psychophysiology Laboratory

Department of Experimental Clinical and Health Psychology

Ghent University

9000 Ghent

Belgium

Tel: +3292648656

Email address: sam.verschooren@ugent.be

CAmerican Psychological Association, 2019. This paper is not the copy of record and may not exactly replicate the authoritative document published in the APA journal. Please do not copy or cite without author's permission. 
1. We demonstrate that the larger cost for switching attention towards internal representations compared to external stimuli cannot be explained in terms of a priming or memory retrieval account.

2. The data seems to be best accounted for by an associative interference account. This account entails that internal attention can be better shielded from external intrusions than the other way around, which raises some intriguing further research questions. 
Abstract

2 At present, the process of switching attention between external stimuli and internal representations is not 3 well understood. To address this, Verschooren and colleagues (2019) recently designed a novel paradigm 4 where participants were cued to switch attention between external and internal information on a trial-by5 trial basis. The authors observed an asymmetrical switch cost, which was larger when switching towards 6 internal than external material, even though participants performed internal trials faster. In the current 7 study, we sought to establish the cause of this asymmetry by adjudicating between predictions from three 8 theoretical accounts: associative interference, priming, and memory retrieval. After replicating the original asymmetry (Experiment 1), we demonstrate that trial-by-trial carryover of attentional settings is not a necessary precondition (Experiment 2). The results from Experiment 3 indicate that the cost asymmetry can be best explained by an associative interference account, against a memory retrieval one. Together, these results therefor provide evidence in favor of an associative interference account and document that shielding attention for internal representations from external intrusions is more efficient than the other way around. This finding advances our understanding of a core aspect of cognitive flexibility and the relationship between external and internal attention. More research on this question and novel ones raised by it is necessary, however. 
2 Attention can be broadly subdivided into two categories by distinguishing between the main substrates it acts upon, i.e. external stimuli versus internal representations (Chun et al., 2011; Myers et al., 2017; Verschooren, Schindler, et al., 2019). Whereas external attention directly depends on currently available

5 perceptual input, internal attention operates on long-term memory (LTM) representations, which are considered to enter working memory (WM) once attention is allocated to them (Oberauer, 2002, 2009). In our interaction with the environment, we often switch attention between both substrates. For example, when we are looking for a grocery item in the store and, after having found it, we retrieve the next item on our memorized grocery list, and hence transit from external to internal attention. Even though the regulation of attention between external stimuli and internal representations is thus clearly central to successful everyday cognition, relatively little is known currently about the mechanisms involved in switching attention to and fro between these modes.

Recently, Verschooren and colleagues (Verschooren, Liefooghe, Brass, \& Pourtois, 2019) validated a novel experimental procedure in which participants randomly switch attention between external stimuli and internal representations on a trial-by-trial basis. After memorizing four figures at the beginning of the experiment, they performed a probe-to-target matching task where the target figure was either presented on screen (external trial) or retrieved from memory based on a location cue (internal trial; see Fig. 1). The trial-to-trial transitions created four conditions of interest: external repetitions, switches towards external, internal repetitions, and switches towards internal. The authors reported a cost for switch trials, with participants being slower and more error prone on switch than repetition trials. Furthermore, this switch cost was clearly asymmetric: it was larger when switching attention to an internal representation after processing an external stimulus than in the reverse direction. Crucially, despite the larger switch cost, participants performed internal trials faster than external trials, thereby ruling out a simple interpretation in terms of asymmetrical task difficulty. 
2 participants switch between a dominant or highly practiced task and a non-dominant one. For example, in the Stroop task (Stroop, 1935), color words are printed in different color fonts (e.g. the word "blue" printed in red) and participant respond either to the name of the word (here: blue) or its color (here: red). When the relevant stimulus dimension on the current trial (e.g. word meaning) is different from that on the previous trial (e.g. color of the word), a cost is observed compared to a task repetition (e.g. two successive word naming trials). Crucially, when switching between (more automatic/dominant) word naming and (less automatic/non-dominant) color naming, participants usually show a larger switch cost when switching towards word naming, despite the overall reaction time advantage on word naming trials (Allport et al., 1994). Two important accounts in the task-switching literature, associative interference and priming, provide competing explanation for this phenomenon. We discuss both in detail below.

It should nonetheless be noted that mapping this pattern onto the findings reported by Verschooren and colleagues (2019) implies that internal attention is more automatic (or dominant) than external attention. However, we know of no other prior empirical evidence supporting this idea, and an advantage for internal over external attention might seem counterintuitive at first sight. Consequently, we cannot take this assumption for granted. Alternatively, it may be the case that external and internal attention are not necessarily imbalanced, but instead, memory retrieval differs between them, and hence this factor would account for this asymmetry. That is, it is usually more difficult to access internal representations (from memory) than external stimuli (perceptually available), but this retrieval cost may be reduced when the internal representations are repeated. If this were the case, a larger internal than external switch cost is expected as well. Even though this account seems to be a priori more plausible, it nonetheless fails to address the origin of the faster mean response times for internal trials.

The aim of the present study was to adjudicate between these three different explanations. The common denominator between these three accounts is that they can all provide an explanation for the 
switch cost asymmetry. In the following, we discuss the two (opposing) task-switching and the memory retrieval accounts, and their mutually exclusive predictions under specific experimental manipulations, separately. We then assess these predictions empirically, to take an important step towards better understanding the processes underlying transitions between external and internal attentional states.

5

\section{Associative interference}

The associative interference hypothesis is an account developed to explain task-switching costs (for reviews, see Monsell, 2003; Vandierendonck, Liefooghe, \& Verbruggen, 2010). It attributes this cost to the activation of task-irrelevant (or competing) memory traces in a task-switching context (e.g., Waszak, Hommel, \& Allport, 2003). These traces, more specifically, are automatically encoded into LTM when engaging with a stimulus, which serves as a retrieval cue during subsequent encounters (Logan, 1988). Retrieval includes the reactivation of the specific attentional settings (e.g., focusing on the ink color versus semantic meaning of the stimulus). Moreover, it has been demonstrated that these traces include abstract control setting as well, such as a flexible versus stable mental state (for recent reviews, see Abrahamse, Braem, Notebaert, \& Verguts, 2016; Braem \& Egner, 2018; Chiu \& Egner, 2017). For example, Chiu and Egner (2017) demonstrated that participants show a decreased switch cost for stimuli that were associated with frequent task switches compared to stimuli associated with low switch frequency. Thus, we similarly assume that an external or internal attentional focus would be encoded with other trial features (i.e., the cues and/or target stimuli), and subsequently retrieved by these features; as a consequence, they could cause interference if retrieved in a situation where the other type of attention is required.

Crucially, interference from the irrelevant attentional setting is especially detrimental on switch trials, as a WM update takes place, making ongoing performance more susceptible to intrusions (Dreisbach \& Haider, 2008, 2009; Dreisbach \& Wenke, 2011). This is in line with an influential model of WM function, which proposes that its content is controlled by a gating mechanism (Frank et al., 2001; 
1 Reilly \& Frank, 2006). The gate is closed when the content is maintained but opened when it needs to be

2 updated. In this state, irrelevant stimulus features (and associated actions and attentional states) can enter

3 WM and have a detrimental effect on task execution. Switch trials are a specific instance of a situation in

4 which a transition from a maintenance to an updating mode is required, and the gate is opened (Mayr et

5 al., 2014). As traces for both competing task sets are retrieved in a task-switching context, they can both

6 access WM and influence behavior. The interference resulting from this competition leads to a switch

7 cost.

8

To account for asymmetrical switch costs, the associative interference hypothesis assumes that dominant and non-dominant task sets differ in the efficiency with which the relevant attentional focus is shielded (Mayr et al., 2014). When switching between a dominant and non-dominant task, the memory traces activating the non-dominant attentional focus are usually not sufficiently strong to interfere on repetition trials of the dominant one. Consequently, robust maintenance prevails. On trials of the nondominant task, on the other hand, the dominant task can interfere with maintenance of the non-dominant task set, even when the task is repeated. On switch trials of both tasks, however, WM needs to be updated with the relevant attentional setting, during which memory traces from the competing task set can interfere equally for the dominant and non-dominant task. The larger switch cost for the dominant task is caused by the difference between robust maintenance on repetition and updating on switch trials.

When applied to the cost asymmetry between external and internal switching, this account entails that the memory traces for their two competing attentional states, i.e., external versus internal attention, are learned associatively when performing the task. On external repetition trials, external attention suffers interference from the internal one. On internal repetition trials, on the other hand, internal attention does not suffer much interference and can be robustly maintained. On switch trials, both attentional states are affecting behavior, resulting in the large internal and smaller external switch cost. 


\section{Priming}

The task priming or "task set inertia" hypothesis was developed to account for task-switching costs as well (Allport et al., 1994; Gilbert \& Shallice, 2002; Yeung \& Monsell, 2003). The key difference with the associative interference hypothesis is that it proposes that task sets are carried over from one trial to the next - often with a recency gradient (Yeung \& Monsell, 2003). That is, the cost is not due to retrieval of competing task sets, but to their positive priming from trial-to-trial, defined as the transient increases in activation for recently performed tasks (but see Allport et al., 1994 for an inhibition account).

This account has similarly been applied to asymmetrical switch costs between dominant and nondominant tasks. Yeung and Monsell (2003) argued that priming of task sets interacts differently with dominant and non-dominant tasks. More specifically, in their model, positive priming is assumed to be especially strong following trials of the non-dominant task (see also Gilbert \& Shallice, 2002). On these trials, control is required to enhance attention to the relevant stimulus features. On trials of the dominant task, on the other hand, attention is more automatically allocated to the dominant stimulus features, without the involvement of additional control (which is assumed to be costly, and therefore minimized when possible). Together, these opposing effects can explain the cost asymmetry: On non-dominant repetition and switch trials, interference from the dominant task set's activation is largely stable. As a result, there is only a small performance difference on both trial types. On dominant trials, on the other hand, the amount of interference from the non-dominant task set differs greatly between switch trials, where control has recently increased the non-dominant task-set's activation, and repetition trials, where its activation has returned to a default low state.

We can again use this account to interpret the asymmetric cost for switches between external and internal attention (Verschooren et al., 2019). According to this account, additional (controlled) activation of external attention on external trials is required, while internal attention is activated more automatically. When switching from an external to an internal trial, the resulting additional activation of external 
attention is primed and results in a large switch cost. On external trials, on the other hand, there is a stable detrimental effect from the more automatic internal attention on both repetition and switch trials. As a result, no large cost emerges.

\section{Retrieval cost for switches to memory}

In contrast to the two previous accounts, we can also formulate an explanation that does not assume an imbalance between external and internal attention. That is, one important difference between external and internal trials is the degree to which they rely on information stored in declarative memory. On internal trials, accessing and retrieving this information can incur an independent cost. Dark (1990) previously argued for such an account after observing a cost asymmetry for switches between perceiving individual letters on screen and retrieving them from memory. Participants performed lists in which letters were either only perceived or only retrieved to performance on lists where a single switch between them occurred. Dark (1990) found a cost only for switches towards memory retrieval, which disappeared when participants were pre-cued and could retrieve the letter in advance. Consequently, she concluded that switches between perception and memory in themselves did not incur a cost, but memory retrieval did (but see Carlson, Wenger, \& Sullivan, 1993; Verschooren et al., 2019 for methodological limitations).

This memory retrieval account can also be applied to interpret the asymmetric switch cost observed in the procedure of Verschooren and colleagues (2019) where external and internal trials were intermixed. That is, we can interpret the larger internal switch cost as resulting from two independently contributing effects: the switch cost itself and a memory retrieval cost. In this interpretation, the presence of the basic switch cost is caused either by associative interference or by priming. In addition, specifically on internal trials, participants need to retrieve the memorized figures from declarative memory. If we further assume that recently refreshed memories are more accessible for retrieval, an additive memory retrieval cost during this switch can explain the larger switch cost for internal trials. This assumption entails that the 
memory retrieval cost will be especially large when switching to internal trials, compared to internal repetitions. In this situation, the internal representations have not recently been refreshed and need to be actively retrieved. In other words, even though there is a similar cost for the switch itself on both external and internal switch trials, an additional memory retrieval cost is present on internal switch trials, which would drive the larger cost observed there.

\section{The current study}

8

The three accounts discussed above, i.e. associative interference, priming, and memory retrieval, have competing predictions under specific experimental settings. These predictions relate specifically to the relative magnitude of the switch costs (i.e., their asymmetry). According to the priming hypothesis, the cost asymmetry will be present only when participants switch between external and internal trials within a mixed block. The associative interference hypothesis, on the other hand, predicts that the asymmetry will be present even in blocks where only external or only internal trials are presented, as long solve a simple mathematical equation. In this design, a priming account predicts no asymmetry, as there 
1 is no additional external activation preceding an internal switch trial, the internal switch cost should be

2 reduced, blunting the interaction effect. The other two accounts do predict a cost asymmetry, as in this

3 design traces for the competing attentional states are present (associative interference) and switches

4 towards internal representations occur (memory retrieval). Finally, Experiment 3 was a between-subjects

5 design, where one group received external trials only and the other internal trials only. Here again,

6 participant performed the mathematical task on a subset of trials. With this design, there is no opportunity

7 to form associations of the competing attentional state, so the associative interference account predicts

8 that the cost asymmetry would be absent in this experiment. More specifically, as the external repetition

9 trials suffer less from the internal interference, the external switch cost should increase. Conversely, as

10 the internal repetition trials never suffered from external interference, the magnitude of the internal switch

11 cost should stay the same. Memory retrieval, on the other hand, assumes that the external switch cost

12 should remain small and the internal one larger, resulting from the (effortful) retrieval operation. To

13 summarize, in Experiment 2 we can compare the predictions from the priming account with the other two

14 accounts. In Experiment 3, associative interference and memory retrieval can be pitted against each other. 


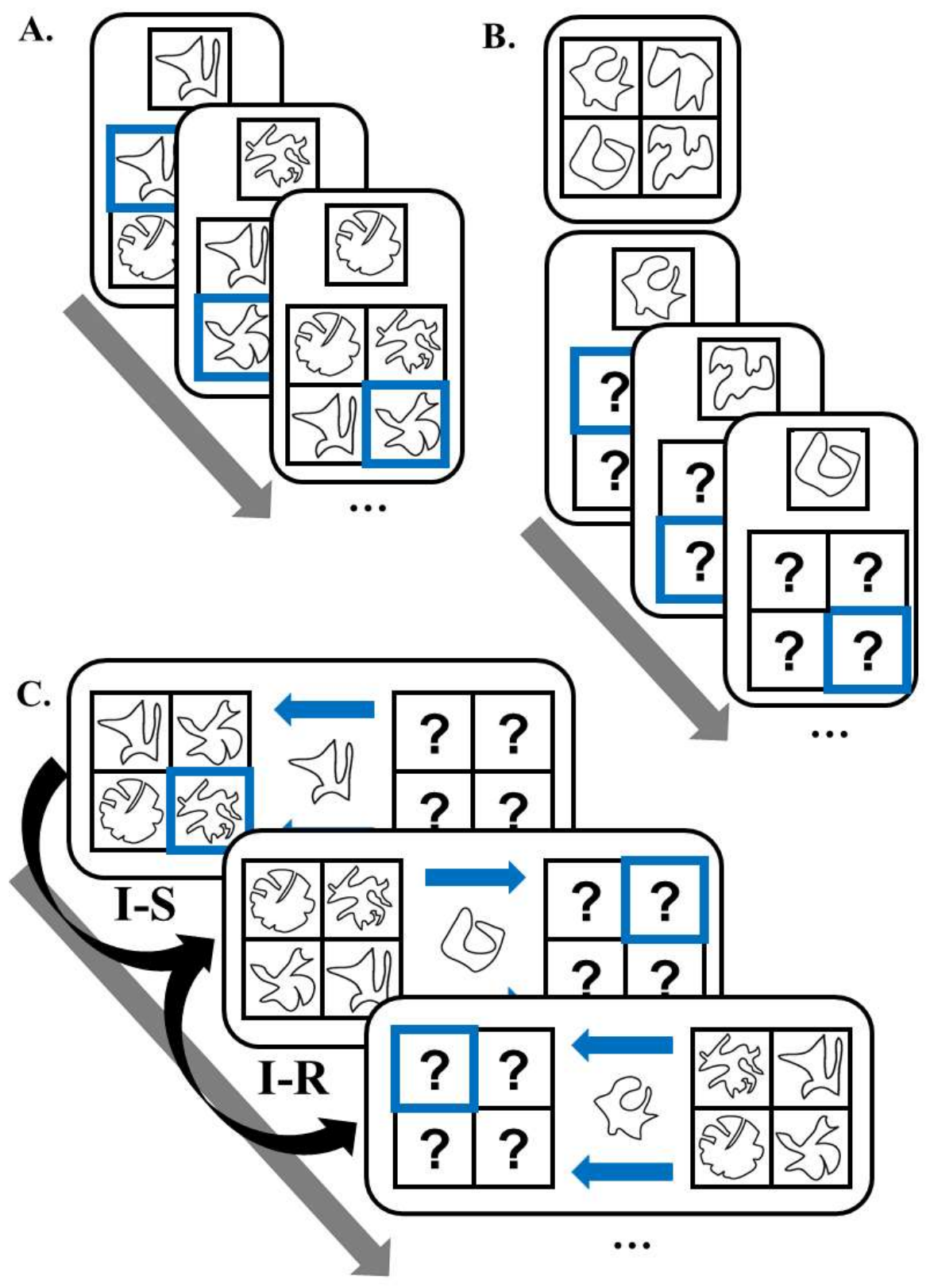

Figure 1. Experimental paradigm. A. External training phase. Participants decided whether the figure above (probe) matched the figure selected in a blue frame, until they reached the accuracy criterion (both >.85 and 4 at least 18 total correct trials). B. Internal training phase. Participants memorized four figures and their locations. 5 On each trial, they decided whether the figure above (probe) was in the selected location (in blue) for the memorized set. The same accuracy criterion was used. C. Experimental task: On each trial, participant decided whether the selected figure (in blue) matched the centrally presented probe. The selected figure was either presented on screen 8 (external trial; first in sequence) or retrieved from the figures memorized in 1B based on the selected location 9 (internal trial; last two). We were interested in the four possible trial-to-trial transitions: External-Repetition (E-R), External-Switch (E-S), Internal-Repetition (I-R), or Internal-Switch (I-S). 


\section{Experiment 1}

\section{Participants}

We recruited 47 participants on Amazon Turk, an online recruiting platform. The sample size was motivated by an a priori power analysis to detect a small effect size (partial $\eta^{2}$ of 0.04 ) in a Repeated Measures Analysis of Variance with $85 \%$ power, which revealed a required sample size of 39 (see also Verschooren et al., 2019). We accepted data submission for participants that performed at $>75 \%{ }^{1}$ accuracy on the experimental task, which resulted in the rejection of six participants (final sample of 41 participants $)^{2}$. Participants signed informed consents and received $\$ 3.3$ for participation.

\section{Methods}

Materials

The experiment was programmed in JavaScript, using the jQuery library (see https://osf.io/t8ery/ for the experiment script). The stimuli were 16 non-verbalizable figures from a larger set of stimuli created by Endo and colleagues (Endo et al., 2003). These black figures were 60 by 60 pixels on a white background screen (900 x 900 pixels). In each run, two sets of four figures were used (one set for the external and another one for the internal trials). The same two sets were used for half of the participants and two other sets for the other half. For each participant, the set used for the internal and external trials was randomized.

\footnotetext{
${ }^{1}$ This criterion was chosen to screen out participants that did not memorize the figures and answered randomly on this part of the task, which could theoretically result in an average accuracy of $75 \%$ (100\% on external trials and $50 \%$ on internal ones).

2 Demographic information for the participants was not saved due to a technical error. Difallah and colleagues (Difallah, Filatova, Ipeirotis, 2018) recently investigated the demographic characteristics of Amazon Turk workers and reported a mean age of approximately 35 years old and an approximately balanced gender ratio. We have found this consistently as well in other (unpublished) experiments with similar sample sizes and in the published literature (e.g. Whitehead \& Egner, 2017). Hence, we operate under the assumption that this describes the current samples.
} 


\section{Procedure}

The experiment began with a training phase, which consisted of an external and internal training task (their order was counterbalanced over participants). The external training task was not strictly necessary to be able to carry out the perceptual trials of the main task, but was included to equate the amount of practice for the external and internal stimuli (see Verschooren et al, 2019, Experiment 2). On each external training trial, a square was presented with one of the external figures in each of its four compartments for 15 s or until response (see Fig. 1A). The locations of these figures were randomized on each trial. One of the four figures would be highlighted in a blue frame, indicating that this figure was the target figure. Simultaneously as well, one of the figures (the probe) would be presented above the square. Participants had to decide whether the target figure was identical to the probe figure. The internal training task began with the presentation of the four figures of the internal set (see Fig. 1B). Participants pressed the space bar after memorizing these figures and their locations to continue to the internal training phase (or after $15 \mathrm{~s}$ had passed). Each internal training trial started with a $350 \mathrm{~ms}$ fixation cross (to remove the after-image of the correct stimulus-location associations presented following an error, see below). Similar to the external training phase, for $15 \mathrm{~s}$ (or until response), a square with four compartments was presented on each trial, with one of them highlighted in a blue frame (the target location) and one of the four figures from the external set above presented above the square (the probe). Different to the perceptual training, however, the compartments of the square were filled with question marks. Participants needed to retrieve the figure that was in the target location from memory and decide whether this figure was identical to the probe figure. If participants made a mistake, the four figures were presented on screen for $5 \mathrm{~s}$ to allow reencoding of the correct stimulus-location associations. Participants moved on to the experiment proper (the practice and main task) once their accuracy was higher than .85 on each of these tasks (and at least 18 trials were performed correctly in total). This criterion was set to assure that all participants memorized the set sufficiently (and equally). 
In the experimental task, each trial started with a $250 \mathrm{~ms}$ fixation cross, after which a square (with

2 four compartments) appeared on either side of it for 300ms (See Fig 1C). One of the squares would contain

3 four figures (perceptual set) and the other one four question marks (cues for LTM set). The locations of 4 these external figures were randomized on each trial. Simultaneously, two arrows, one above and one 5 below the fixation cross, would point to the relevant square for that trial (figures and question marks on 6 perceptual and memory trials, respectively). At the same time, one of the four compartments in this square 7 would be highlighted in a blue frame, indicating that this was the target that needed to be matched to the 8 probe. On external trials, the target figure was presented on screen. On internal trials, participants needed to use the target location as a cue to retrieve the figure previously memorized in this location (see next paragraph). Finally, after this $300 \mathrm{~ms}$ window, a central probe replaced the fixation cross (all other stimuli remained on screen), which needed to be compared by the participants to the target. This final screen was presented for $2500 \mathrm{~ms}$ or until response.

For both the training tasks and experimental task, participants responded with the "d"-key if the target and probe were identical and "k"-key if they were not. This response mapping was counterbalanced over participants. Participants received feedback after each trial of the training tasks and after each trial of the practice block of the experimental task. After each block, they received feedback on their accuracy in that block. there were 2 warm-up trials and 80 experimental trials. Trial order was counterbalanced within each bock to create an equal number of trials in each of the four conditions. In addition, for each of these conditions, there was an equal number of match and mismatch trials (probe-target compatibility). On each trial, there was a .50 chance for the perceptual figures to appear on the left or right side and a .25 chance for each of the four locations to be the target (immediate repetitions of target position or probe were excluded). The 
order of the nine blocks was randomized for each participant, but the trial sequence within these blocks was pre-defined to meet the above restrictions.

3

\section{Design}

The task formed a repeated-measures 2 (Modality: external, internal) x 2 (Switch: repeat, switch) factorial design. The figures were presented on screen and retrieved from memory on external and internal trials, respectively. On repetition trials, the Modality of the current trial was the same as that on the preceding trial versus different on switch trials. This design resulted in four conditions: ExternalRepetition (Ext-Rep), External-Switch (Ext-Swi), Internal-Repetition (Int-Rep), and Internal-Switch (IntSwi). The main dependent variables were the response times (RT) and error rates (ER) in each condition.

\section{Data Analysis}

The raw data and analysis scripts can be found online (https://osf.io/t8ery/). We removed the practice trials, the trials of the external and internal training phase, and the first two trials from each experimental block. In addition, we removed trials on which an error was made and those preceded by an error. The data were preprocessed and visualized using the tidyverse (Wickham, 2017) and ggplot2 (Wickham, 2016) libraries in R (R core team, 2018; version 1.1.456).

Participant had very low error rates (ER; sample mean around .05 in the least accurate condition) and there was no evidence for a speed-accuracy trade-off (see Table 1), so we focused the analysis on response times (RT). We analyzed the data with a Bayesian generalized linear multilevel model (BGLMM) approach, using the brms (Bürkner, 2017) and emmeans (Lenth, 2018) libraries. When there are repeated observations for each participant, a linear multilevel model is preferable over ordinary linear regression, as it considers variability at different levels of the dataset and allows them to inform each other (McElreath, 2016; Nalborczyk et al., 2018). Moreover, it is advisable to use a GLMM when analyzing RT data, which are rarely normally distributed and often show a skewed distribution (Lo \& Andrews, 2015; 
1 Speelman \& McGann, 2013). We opted for a GLMM with an inverse Gaussian with natural logarithmic

2 function, which we previously found to describe RT data on this type of protocol best (Verschooren, 3 Liefooghe, et al., 2019). Finally, we specified the model using a full random structure, as has been 4 recommended in the literature (see Barr, Levy, Scheepers, \& Tily, 2013). That is, in addition to estimating 5 the group-level effects for Modality, Switch, and their interaction, we estimated these effects for each 6 participant as well.

Within this model, we then looked at the presence or absence of the fixed effects of interest. We 8 provide the model summary (fixed effects) and planned contrasts, which give the model estimate of the switch cost for external and internal trials. As the model estimates are not in the response scale, we backtransformed them using the emmeans package. For all effects, we present the $95 \%$ high probability density interval (HPDI) of the posterior distribution.

We analyzed the data in a Bayesian framework because our critical test consisted of detecting evidence for the presence or absence of the interaction effect. Within a Bayesian framework, evidence in favor of or against the null-hypothesis can be obtained in the form of a Bayes Factor (BF). We obtained BFs using the Savage-Dickey density ratio method from the brms package (see Wagenmakers et al., 2010). This method is very sensitive to the specific prior set by the researcher, so we used weakly informative priors on the fixed effects (a normal distribution centered around 0 with a standard deviation of 0.03 ). The BF testing for evidence in favor of or against the null hypothesis then represents the amount of posterior updating after the data are fit by the model. In addition to these BFs, we use a region of practical equivalence (ROPE), which is less reliant on priors. Kruschke (2018) suggests to set the width of the ROPE to -0.1 to 0.1 times the standard deviation of the posterior distribution, corresponding to a negligible effect size. We used 0.2 as a more conservative estimate, but this cutoff did not influence the results. If the $89 \%$ CI falls completely outside the ROPE, we can conclude that we have a non-negligible effect. 
Table 1

Reaction times and error rates for Experiment 1-3

\begin{tabular}{|c|c|c|c|c|c|c|}
\hline Exp. & Src. & $\mathrm{Seq}$ & $R T(S D)$ & $E R(S D)$ & $R T$ Cost & ER Cost \\
\hline \multirow{4}{*}{1} & \multirow{2}{*}{ Ext } & Rep & $815(165)$ & $.03(.04)$ & \multirow{2}{*}{36} & \multirow{2}{*}{.01} \\
\hline & & Swi & 851 (177) & $.04(.05)$ & & \\
\hline & \multirow{2}{*}{ Int } & Rep & $912(204)$ & $.07(.06)$ & \multirow{2}{*}{59} & \multirow{2}{*}{.01} \\
\hline & & Swi & $971(212)$ & $.08(.06)$ & & \\
\hline \multirow{4}{*}{2} & \multirow[t]{2}{*}{ Ext } & Rep & 744 (113) & $.03(.03)$ & \multirow[t]{2}{*}{52} & \multirow[t]{2}{*}{.00} \\
\hline & & Swi & 796 (136) & $.03(.03)$ & & \\
\hline & \multirow{2}{*}{ Int } & Rep & $875(154)$ & $.05(.04)$ & \multirow{2}{*}{80} & \multirow{2}{*}{.01} \\
\hline & & Swi & $955(184)$ & $.06(.05)$ & & \\
\hline \multirow{4}{*}{3} & \multirow[t]{2}{*}{ Ext } & Rep & $822(176)$ & $.03(.04)$ & \multirow[t]{2}{*}{62} & \multirow[t]{2}{*}{.01} \\
\hline & & Swi & 884 (194) & $.04(.04)$ & & \\
\hline & \multirow{2}{*}{ Int } & Rep & 885 (194) & $.05(.06)$ & \multirow{2}{*}{72} & \multirow{2}{*}{.01} \\
\hline & & Swi & 957 (209) & $.06(.07)$ & & \\
\hline
\end{tabular}

1

2 

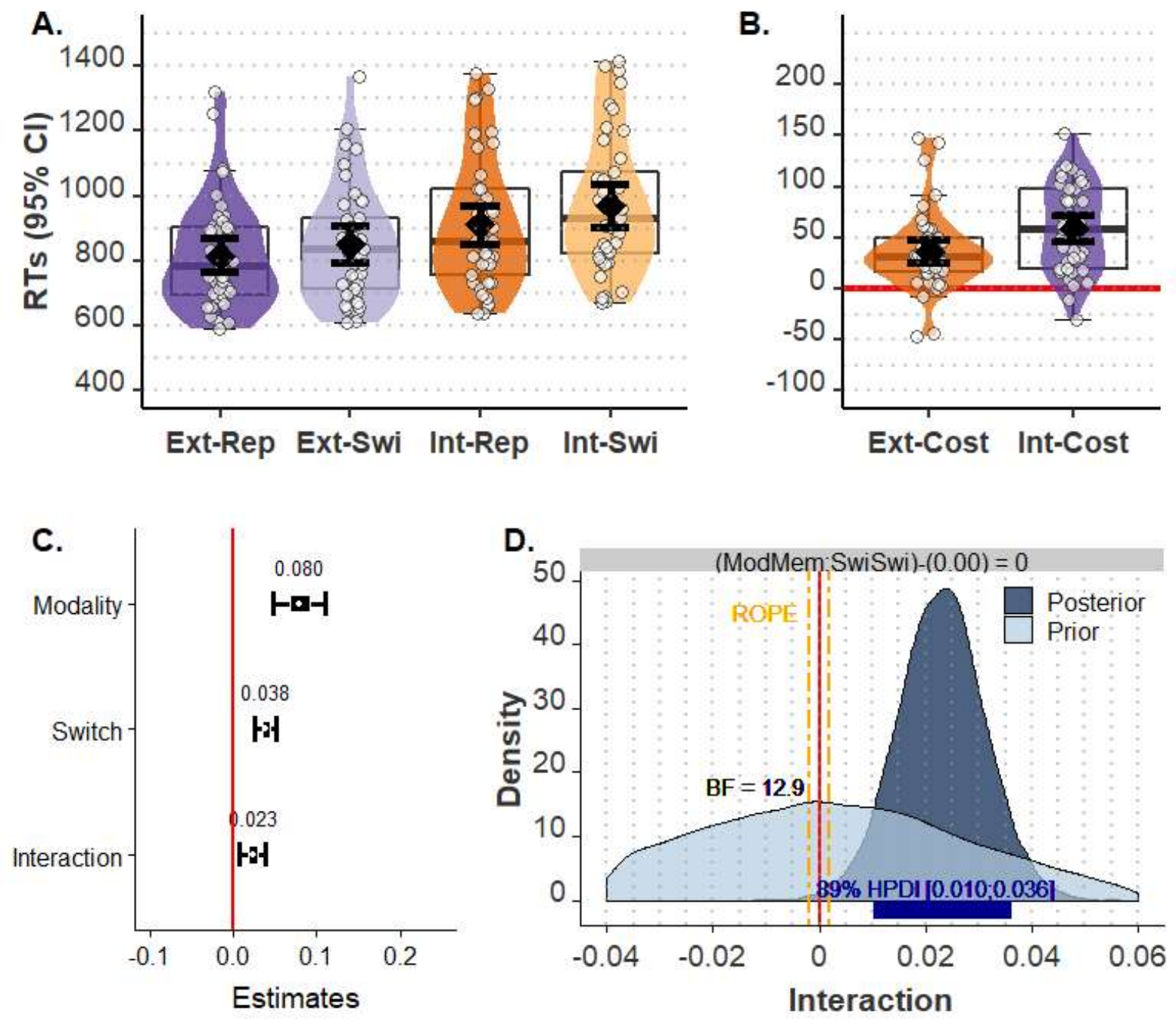

Figure 2. Response times for Experiment 1. In black: Mean response time and 95\% confidence interval (CI). In white: individual participants' means. A. External-Repetition (E-R), External-Switch (Ext-Swi), Internal-Repetition (Int-Rep) and Internal-Switch (Int-Swi) condition. B. Switch cost for External (ExtSwi $>$ Ext-Rep) and Internal (Int-Swi $>$ Int-Rep). C. The (transformed) model estimates for the fixed effects. The thick line and error bars represent the 55\% and 95\% HPDI, respectively. D. The prior (light blue) and posterior (dark blue) distribution for the interaction effect. The BF (12.9) represents their ratio for the hypothesis that there is an interaction effect. The ROPE (orange) and 89\% HDPI (blue) do not overlap (see Krusche, 2018).

The model summary revealed a main effect for Modality $(\beta=0.079,95 \%$ HPDI $=[0.046 ; 0.109])$ and for Switch $(\beta=0.038,95 \% H P D I=[0.025 ; 0.051]$; see Fig. 2C). More specifically, participants were slower on internal than external trials and on switch than repetition trials (see Fig. 2A). Crucially, the interaction effect was present as well, providing evidence for the switch cost asymmetry $(\beta=0.023,95 \%$ 
$H P D I=[0.007 ; 0.039]$; see Fig. 2B-C). Converted back to the response scale, the model estimated the

2 intercepts of the conditions to be $805 \mathrm{~ms}(95 \% H P D I=[754 ; 860]), 836 \mathrm{~ms}(95 \% H D I=[781 ; 893]), 871 \mathrm{~ms}$ $(95 \% \mathrm{HPDI}=[811 ; 933])$, and 926ms $(95 \% \mathrm{HPDI}=[862 ; 993])$ for Ext-Rep, Ext-Swi, Int-Rep, and IntSwi, respectively. Planned contrasts for the effect of Switch in both Modalities revealed that the switch cost was indeed asymmetric in the expected direction, with a smaller cost when switching attention to external stimuli $(31 \mathrm{~ms}, 95 \% H P D I=[21 ; 41])$ than to internal representations $(55 \mathrm{~ms}, 95 \%$ HPDI $=[42 ; 68]$; see Fig. 2). Crucially, when we look at the BF (12.9), we can conclude that we have strong evidence in favor of the presence of an interaction effect (Kass \& Raftery, 1995; see Fig. 2D). This agrees with the evidence gathered through the ROPE as well, which falls completely outside the 89\% HPDI (see Fig. 2D).

\section{Discussion}

In Experiment 1, participants responded more slowly when the modality (external versus internal)

of the current trial was different than the modality of the previous one, compared to a repetition. Moreover, the model estimated this cost as larger when switching towards internal $(55 \mathrm{~ms})$ than external $(31 \mathrm{~ms})$ trials. In other words, we replicated the asymmetric cost $(\mathrm{BF}=12.9)$ for these switches reported in Verschooren and colleagues (2019). ${ }^{3}$

3 This replication was successful despite profound differences in the sample pool characteristics (Amazon Turk participants primarily from USA and India versus undergraduate psychology students from Belgium) and minor changes in the experimental protocol (most notably the amount and presentation of the training phases and the order of the target-probe appearance, see Verschooren, Liefooghe, Brass, \& Pourtois, 2019). This further demonstrates the robustness of this effect. 
1 asymmetry will be present whenever participants switch towards an internal trial. In Experiment 2, we

2 pitted the priming hypothesis against the other two hypotheses by evaluating whether trial-to-trial 3 carryover of attentional settings is a necessary condition for the cost asymmetry to occur. More 4 specifically, in Experiment 2, participants carried out pure blocks of either perceptual or memory trials 5 and were occasionally interrupted by a third, independent task in which they solved simple math equations.

6 If we were to observe larger costs in the internal blocks than perceptual blocks, we would be able to infer 7 that carryover of attentional settings from one modality to the other is not a necessary condition for the 8 asymmetry. 


\section{Experiment 2}

\section{Participants}

54 participants were recruited through Amazon Turk (see power analysis Experiment 1). We rejected nine participants with accuracy lower than 75\% (45 included). Participants signed informed

5 consent and received $\$ 3.3$ for participation.

6

7

8

9

\section{Methods}

Materials

\section{Procedure}

Materials were the same as in Experiment 1 (see https://osf.io/t8ery/ for the experiment script).

For the interrupting task, we used a list of simple mathematical equations (Vassena et al., 2014).

The training task and experimental trials were identical to Experiment 1. However, on one in four experimental trials, a simple mathematical equation followed the probe-to-target matching. These trials consisted of a single screen in which three digits needed to be added (“ $\mathrm{A}+\mathrm{B}+\mathrm{C}=$ ?”). On each trial, one correct and one incorrect solution was provided ("Press the ' $\mathrm{d}$ '-key if the correct answer is $\mathrm{X}$ and press the ' $k$ '-key if the correct answer is $\mathrm{Y}$ '). The correct result never exceeded 9 and the incorrect answer differed by 1 or 2 from the correct one. This interrupting task was taken from earlier research addressing a similar question (Mayer et al., 2014) and arguably relies on both external and internal attention.

Participants responded with the "d"- and "k"-key on all trials (counterbalanced over participants), but there was no consistent response mapping for correct and incorrect trials on the interrupting task. That is, on each of these trials two response options were provided: "Press ' $d$ '-key if answer is $\mathrm{X}$ and ' $\mathrm{k}$ ' is answer is $\mathrm{Y}, ”$ and the correct response (X versus $\mathrm{Y}$ ) differed from trial-to-trial. 
The same independent variables were used as in Experiment 1 (Modality and Switch), but Modality was alternated from block-to-block instead of trial-to-trial. For the Switch variable, switch trials were those preceded by the interrupting task and repetition trials those that were not. The dependent variables were RT and ER in the different conditions.

The experiment consisted of two practice blocks (one external and one internal) and eight experimental blocks (four external and four internal). The block order alternated between external and internal, but their sequence was counterbalanced over participants (A-B versus B-A). Each trial had a .25 chance to be followed by the interrupting task (160 experimental trials). Within each block, there was an equal number of trials following the interruption form each Modality (10 per Modality per block). For these trials, there was an equal number of match and mismatch responses for each Modality. Side of presentation, probe identity, and target location were randomized as in Experiment 1.

The training tasks were the same as in Experiment 1 and were provided two times for each block type (i.e. once before the practice block and first experimental block for each Modality). removed. The same BGLMM analysis with a full random model was used. 

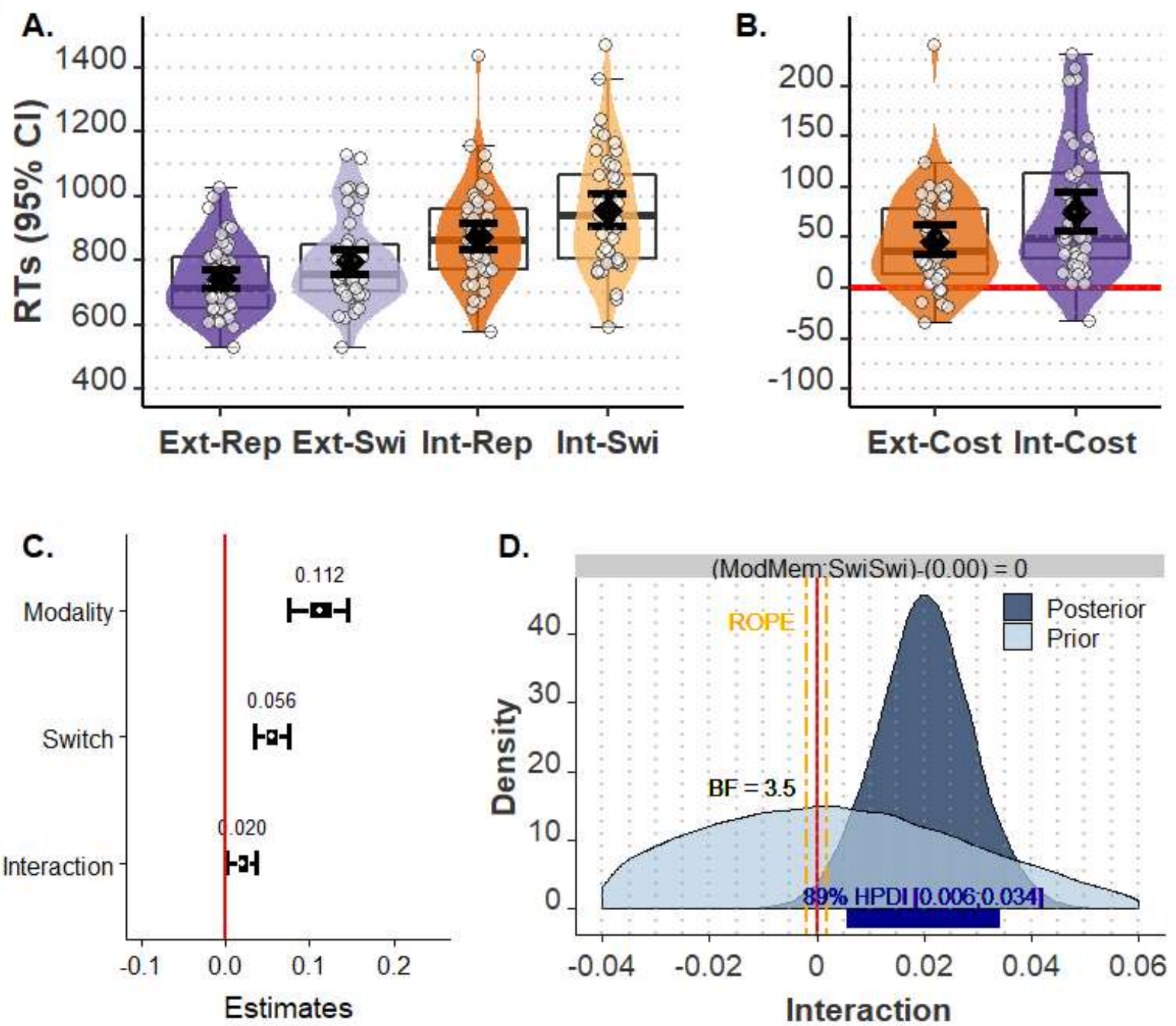

Figure 3. Response times for Experiment 2. In black: Mean response time and 95\% confidence interval (CI). In white: individual participants' means. A. Ext-Rep, Ext-Swi, Int-Rep and Int-Swi) condition. B. Switch cost for External (Ext-Swi $>$ Ext-Rep) and Internal (Int-Swi $>$ Int-Rep). C. The (transformed) model estimates for the fixed effects. The thick line and error bars represent the 55\% and 95\% HPDI, respectively. D. The prior (light blue) and posterior (dark blue) distribution for the interaction effect. The BF (3.5) represents their ratio for the hypothesis that there is an interaction effect. The ROPE (orange) and $89 \%$ HDPI (blue) do not overlap (see Krusche, 2018).

The model summary revealed a main effect for Modality $(\beta=0.111,95 \%$ HPDI $=[0.074 ; 0.144])$ and for Switch $(\beta=0.055,95 \%$ HPDI $=[0.0 .034 ; 0.075]$; see Fig. 3C), with participants being slower on internal and switch trials compared to external and repetition trials, respectively (see Fig. 3A). In addition, an interaction effect was present as well $(\beta=0.020,95 \%$ HPDI $=[0.002 ; 0.038]$, see Fig. 3B-C). The 
model estimated the intercepts for Ext-Rep, Ext-Swi, Int-Rep, and Int-Swi to be $743 \mathrm{~ms}(95 \%$ HPDI= $[708 ; 780]), 786 \mathrm{~ms}(95 \% H P D I=[744 ; 828]), 831 \mathrm{~ms}(95 \% H P D I=[784 ; 877])$, and $897 \mathrm{~ms}(95 \% H P D I=$ $[839 ; 951])$, respectively. Planned contrasts revealed that the switch cost was indeed asymmetric in the expected direction, with a smaller cost to switch towards external trials $(42 \mathrm{~ms}, 95 \%$ HPDI= $[27 ; 57])$ than to internal trials $(65 \mathrm{~ms}, 95 \% \mathrm{HPDI}=[46 ; 82])$. From the BF $(3.5)$, we can conclude that there is substantial evidence in favor of the interaction effect (Kass \& Raftery, 1995; see Fig. 3D). Here again, this conclusion is further corroborated by the lack of overlap between the ROPE and the 89\% HPDI (see Fig. 3D).

\section{Discussion}

In Experiment 2, we tested the competing predictions of the priming hypothesis versus the associative interference and memory retrieval hypotheses. Participants switched between external and internal blocks and were occasionally interrupted to solve a mathematical equation. The priming hypothesis predicted no switch cost asymmetry in this situation, as there was no opportunity for trial-bytrial carryover of attentional settings from external to internal and vice versa. The associative interference and memory retrieval hypotheses, in contrast, both predicted the presence of a cost asymmetry (see Introduction). We found clear statistical evidence for the latter result, with a larger switch cost for internal (80ms) than external $(52 \mathrm{~ms})$ trials. Even though the evidence is less strong compared to Experiment 1, it is still substantial however $(\mathrm{BF}=3.5)$. Therefore, we can reasonably exclude the priming account as a viable explanation for this asymmetry.

However, we cannot yet distinguish between the other two competing accounts, as both had their prediction of the presence of an interaction effect confirmed in Experiment 2. Under certain experimental settings, they nonetheless have opposing predictions. The associative interference hypothesis proposes that the cost asymmetry results from worse shielding against competing attentional states on Ext-Rep than Int-Rep trials. In other words, if there is no opportunity to learn these competing attentional states, the 
1 asymmetry should disappear. The memory retrieval hypothesis, on the other hand, predicts that switching 2 towards internal representations will always (e.g., even though a competing attentional state, here external, 3 is not activated) be more costly than switching to external stimuli. To adjudicate between these two 4 accounts, we used a between-subjects design in Experiment 3 (with one group of participants that 5 performed solely external blocks and the other one solely internal blocks) because it allowed us to explore 6 the behavior of the switch cost under pure experimental conditions, i.e., participants in each group had no 7 experience whatsoever with the other attentional set and thus, a modulation of the switch cost across the 8 two main conditions/groups could not be explained by the presence of this competing attentional set. If 9 we were to find no cost asymmetry in Experiment 3, we would be able to reject the memory retrieval 10 hypothesis. Furthermore, the associative interference hypothesis predicts that the external switch cost will 11 actually become larger in this situation than Experiment 1 because participants could better shield external 12 attention on Ext-Rep trials (i.e. faster RTs on Ext-Rep trials). In other words, if the asymmetry were to 13 disappear because the external cost increases, this would be considered strong evidence in support of the 14 associative interference hypothesis. 


\section{Experiment 3}

\section{Participants}

We recruited 91 participants on Amazon Turk, 47 and 44 for the external and internal condition, respectively. This sample size was based on an a priori power analysis in $\mathrm{G}^{*}$ Power to find a small effect size (partial $\eta^{2}$ of 0.03 ) in a RM ANOVA with a within-between interaction with $85 \%$ power, which revealed a required sample size of 76 participants. Six were excluded in the external condition and three in the internal conditions, resulting in 41 participants in each condition. Participants signed informed consent and received $\$ 3.3$ for participation.

\footnotetext{
Methods

Materials, procedure, and design

The materials from Experiment 2 were also used in Experiment 3. The design from Experiment 2 was adapted to a between-subjects one, but was otherwise identical. In the external condition, participants carried out one practice block and eight experimental blocks of the external trials. Before the practice block and first block, they received the external training task. The participants in the internal condition did the same for the internal trials and the internal training task.
}

\section{Data Analysis}

The data preprocessing and analysis was identical as in Experiment 2 (see https://osf.io/t8ery/).

\section{Results}



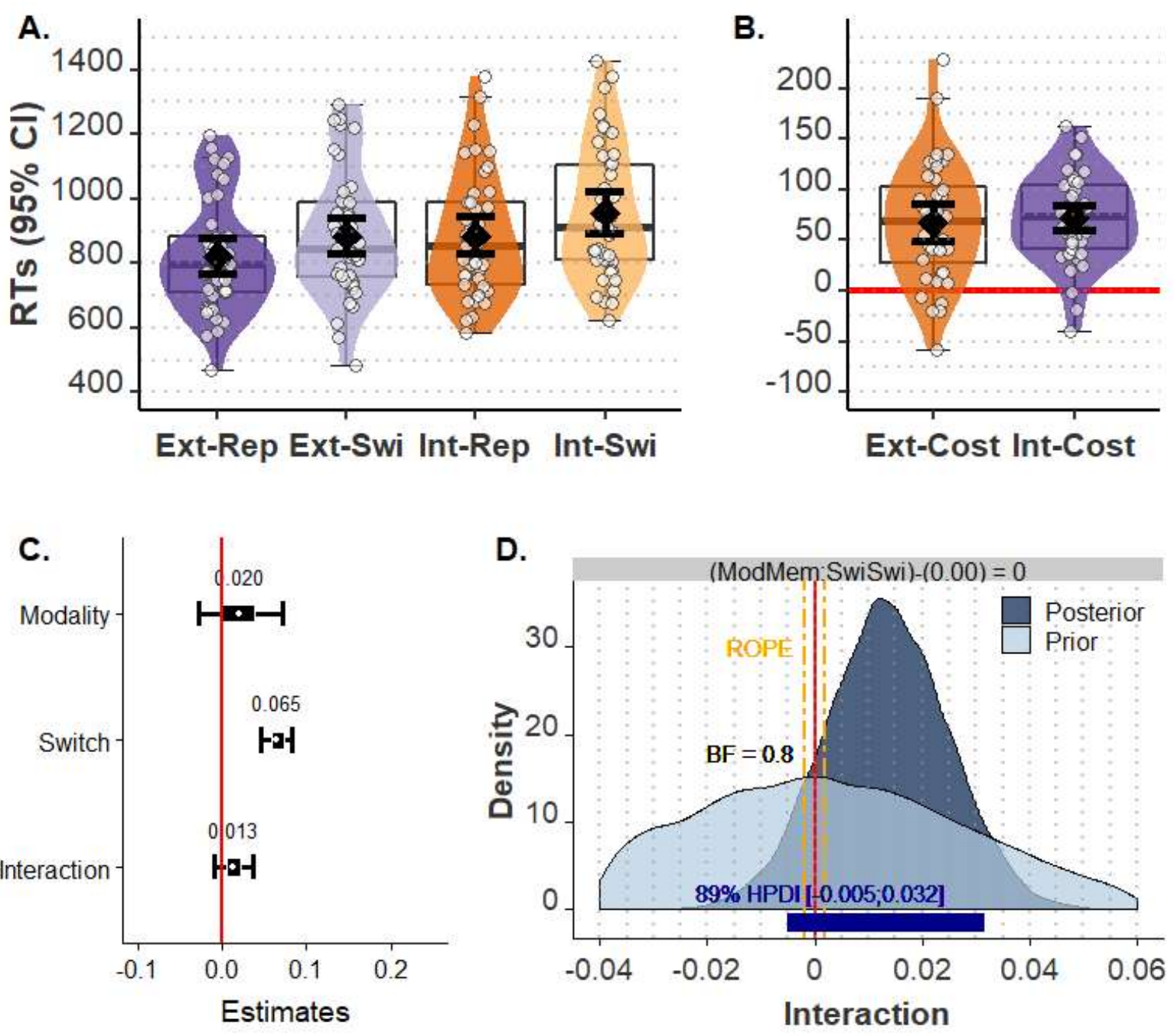

Figure 4. Response times for Experiment 3. In black: Mean response time and 95\% confidence interval (CI). In white: individual participants' means. A. Ext-Rep, Ext-Swi, Int-Rep and Int-Swi condition. B. Switch cost for External (Ext-Swi $>$ Ext-Rep) and Internal (Int-Swi > Int-Rep). C. The (transformed) model estimates for the fixed effects. The thick line and error bars represent the $55 \%$ and $96 \%$ HPDI, respectively. D. The prior (light blue) and posterior (dark blue) distribution for the interaction effect. The $\mathrm{BF}(0.8)$ represents their ratio for the hypothesis that there is an interaction effect. The ROPE (orange) and $89 \%$ HDPI (blue) do not overlap (see Krusche, 2018).

The model showed a significant main effect for Switch $(\beta=0.065,95 \% H D I=[0.046 ; 0.083]$, see Fig. 4C), with participants being slower on switch than repetition trials (see Fig 4A). The effect for Modality $(\beta=0.020,95 \% H D I=[-0.030 ; 0.070])$ and the interaction between these two factors $(\beta=0.013$, 95\% HDI $=[-0.010 ; 0.036]$ ) were not present, however (see Fig. 4A-C). The model estimated the intercepts at $826 \mathrm{~ms}(95 \% \mathrm{HDI}=[780 ; 870]), 881 \mathrm{~ms}(95 \% \mathrm{HDI}=[831 ; 932]), 843 \mathrm{~ms}(95 \% \mathrm{HDI}=[796 ; 889])$, and 
$1911 \mathrm{~ms}(95 \% H D I=[859 ; 962])$ for the Ext-Rep, Ext-Swi, Int-Rep, and Int-Swi trials. The external switch

2 cost was estimated at $56 \mathrm{~ms}(95 \% H D I=[40 ; 71])$ and the internal one at $68 \mathrm{~ms}(95 \% H D I=[56 ; 81])$.

3 Crucially, compared to Experiments 1 and 2, the BF (0.8) changes direction and no longer provides

4 evidence in favor of the presence of an interaction (see Fig. 4D). This is confirmed by the ROPE, which

5 falls completely within the $89 \%$ HPDI. The evidence in favor of the absence of an interaction is not

6 conclusive, however.

\section{Discussion}

9 $17=0.8)$.

Experiment 3 was designed to adjudicate between rival predictions of the associative interference and memory retrieval hypotheses. To this aim, a between-subjects experimental design was used. According to the associative interference account, no cost asymmetry should emerge, as there was no opportunity to learn (and subsequently retrieve) competing attentional states. The memory retrieval account, on the other hand, did predict an asymmetry, as switching towards memory should always be more costly than switching towards perception. Whereas we again found a robust switch cost in Experiment 3, this cost was no longer asymmetric. However, the current data do not allow us to claim evidence in favor of the absence of the interaction, even though the evidence is going in that direction (BF

Notwithstanding this caveat, two additional pieces of evidence in favor of an associative interference account are worth noting here. First, the cost on external trials actually increased in this experiment compared to the two previous ones (see Fig. 5). This was confirmed by two-sided independent samples T-Tests, which showed that there was no difference between the magnitude of the external switch cost in Experiment 1 and $2\left(31 \mathrm{~ms}\right.$ vs. $42 \mathrm{~ms} ; T_{84}=-1.35, p=0.18$, Cohen's $\left.d=-0.29\right)$. This cost was statistically different between Experiment 1 and Experiment 3 (31ms vs. 56ms; $T_{80}=-3.79, p<0.001$, Cohen's $d=-0.84$ ). Between Experiment 2 and 3 it was numerically different and trending towards 
1 significance (42ms vs. $56 \mathrm{~ms} ; T_{84}=-1.79, p=0.07$, Cohen's $d=-0.39$ ). This is a counterintuitive finding

2 that was predicted exclusively by the associative interference hypothesis, i.e. it naturally follows from the 3 more efficient shielding on Ext-Rep trials when no competing attentional states are stored. The memory 4 retrieval account, on the other hand, would predict that the external (and internal) switch costs are 5 equivalent in all experiments, which is not what we observed. Second, there were no differences in the magnitude of the internal switch costs in Experiment 1-3

7 (Ts $<1.62, p s>0.11)$. This finding serves as an argument against an alternative interpretation at the 8 methodological level. That is, one might argue that an important remaining difference between 9 Experiment 1 and 2, on the one hand, and Experiment 3, on the other, is that participants in the internal 10 condition of Experiment 1 and 2 sometimes needed to attend to the external stimuli. This in turn might 11 have disrupted the stable maintenance of the internal representations and resulted in an increased internal retrieval cost when switching towards them (as the memory retrieval account predicts as well). In Experiment 3, participants did not have to attend the external stimuli, so, following this reasoning, internal maintenance would not have suffered. This would reduce the internal switch cost in Experiment 3, blunting the interaction effect. However, what we actually observe is that the internal switch cost stays the same and external one increases. Here again, the associative interference account predicts that the internal cost should not be affected by the presence or absence of external trials. Overall, we therefore conclude that the statistical evidence gathered in Experiment 3 favors the associative interference over the memory retrieval account. 
In this series of experiments, we aimed to contrast three rival accounts for the finding that even

though participants are faster on internal than external attention trials, they show a larger cost when switching attention to internal representations than to external stimuli. These accounts, i.e. associative interference, priming, and memory retrieval, make different predictions under specific experimental settings. More specifically, the priming hypothesis predicts a larger cost for switching attention to internal representations than to external stimuli only when an internal trial is preceded by an external trial, and vice versa. The associative interference account, on the other hand, hypothesizes that the asymmetry occurs in updating situations in which associations have been previously learned between bottom-up stimulus features and competing (external and internal) attentional settings for that stimulus. According to this account, if there are no competing traces, then no cost asymmetry arises. Finally, the memory retrieval account predicts that the cost to switch to an internal representation will systematically be larger than that to switch to an external stimulus, as an additive memory retrieval process takes place, and this, irrespective of the presence or absence of a competing attentional set. Crucially, whereas the first two accounts rely on the assumption that there is an imbalance between external and internal attention, the third one does not. We pitted these predictions against each other in three experiments and found that the data could be best explained in terms of an associative interference account. Even though we are cautious in drawing definite conclusions on the basis of these data alone, these novel findings raise some intriguing research questions, as discussed below.

In Experiment 1, we attempted to replicate using an online experiment (by means of Amazon Mechanical Turk) as opposed to a lab experiment (Verschooren et al., 2019), the cost asymmetry found previously when participant switched randomly on a trial-by-trial basis between external and internal trials in mixed blocks. We found that participants show a cost when switching between external stimuli and internal representations and that this cost was indeed larger for switching attention towards internal 
representations (model estimate: 55ms) than towards external stimuli (31ms; see Fig. 5A), thereby closely replicating Verschooren and colleagues (2019). Experiment 2 was designed to arbitrate between the associative interference and memory retrieval hypotheses on the one hand and the priming hypothesis on the other. All participants alternated between pure blocks of either external or internal trials and solved a simple mathematical equation on a subset of trials. We found a switch cost asymmetry following this interruption that was estimated at $65 \mathrm{~ms}$ when switching towards an internal representation and $42 \mathrm{~ms}$ when switching towards an external stimulus (see Fig. 5B). This finding excludes priming as a plausible explanation of the cost asymmetry, as trial-by-trial switching is a necessary condition for this account. Finally, Experiment 3 was used to disentangle the associative interference from the memory retrieval account. Half of the participants were exclusively confronted with external trials while the other half exclusively with internal trials, a between-subjects manipulation which ensured that external and internal attentional settings did not compete or interfere with each other. Here again, participants solved simple mathematical equations on a subset of the trials. Unlike Experiments 1 and 2, we found no statistical evidence for a cost asymmetry following the interruptions in this experiment (the switch cost was estimated at $68 \mathrm{~ms}$ and $56 \mathrm{~ms}$ for internal and external trials, respectively; see Fig. 5C), which allows us to exclude memory retrieval as a viable explanation for the asymmetry. However, even though the BF reversed direction towards favoring the null, the evidence provided by it was not conclusive $(\mathrm{BF}=0.8)$. That being said, the associative interference account also predicted that the external switch cost would become larger in this single task design (compared to situations where the other task had also been experienced, as in Experiments 1 and 2), which is precisely what we observed (see Fig. 5). That is, according to this account, the smaller cost in a mixed task design is not due to the fact that switching towards external stimuli might be easier, but instead to less efficient (or more difficult) shielding on external repetition trials. In a single task design, there are no competing attentional states that can trigger updating attempts during this inefficient shielding, but the RTs on switch trials are not affected (as 
updating is still required here). This increased cost is not something one would intuitively expect in a single task setting otherwise, as one would expect that it is less effortful to return to the main task. In addition, the internal switch cost was not smaller in Experiment 3 than in Experiment 1 and 2, which excludes further alternative explanations in terms of a disruption of internal maintenance by the external stimuli (see Discussion Experiment 3).

Taken together, these results therefore provide strong evidence against a priming and some evidence against a memory retrieval account, and for the associative interference hypothesis. To recapitulate (see Introduction), associative interference proposes that three conditions need to be met for a cost asymmetry to arise: (i) memory traces for both competing attentional states have been paired to stimulus features and are automatically retrieved when the stimulus is encountered; (ii) the WM gate is opened when transitioning to an updating mode, allowing both traces to simultaneously enter; (iii) when the gate is closed, the more dominant attentional state can efficiently shield against interference from the other, but not the other way around. When these three conditions are met, the cost asymmetry results from the large difference between efficient shielding on repetition trials and inefficient shielding on switch trials for one task and the inefficient shielding on both repetition and switch trials for the other. In the paradigm used here, the actual interference is likely driven by the associative encoding of an external and internal attentional focus with a certain spatial location in the stimulus array, as the same locations are used on external and internal trials. Subsequently, when such a location is selected on a trial, both attentional foci are activated and (asymmetrically) interfere with one another.

One observation nonetheless deserves further attention. Our theoretical framing in the introduction was strongly influenced by the findings presented in Verschooren and colleagues (2019). These authors observed a larger cost for switches towards internal representations, but faster RTs on internal trials in general. This pattern of results suggested the existence of dominant vs. non-dominant attentional sets (see Introduction section) and partly motivated the current study. In the series of experiments reported here, 
1 however, we did not find a general RT benefit for internal trials - even though the cost asymmetry

2 remained. As a matter of fact, participants were faster on external trials in Experiments 1 and 2. This might

3 be due to a reduction in the number of training phases administered to the participants. Whereas a (external

4 and internal) training phase was administered before each block in Verschooren et al. (2019), here we did

5 it only before the practice and first block. As the internal training is arguably more task-relevant, given

6 the importance of the learned stimulus-location associations for performing the task, compared to mere

7 stimulus familiarization in the external case, this may have affected the response speed of the participants

8 (in general). Hence, although training could potentially influence performance (especially on internal

9 trials), it appears that this effect can be dissociated from that related to switches between external and internal attention, likely caused by associative interference. The asymmetry we observe, then, might be more inherent and stem from the enhanced shielding of internal compared to external attention, as our new results suggest. In this context, it should also be noted that this main effect (general response speed difference between internal and external conditions) was never of theoretical interest: the common denominator between the three different theoretical accounts considered in our work was their potential to predict the presence or absence in of the cost asymmetry in different experimental contexts. As stated in the introduction, we did not assume internal dominance because evidence in favor of it is lacking in the extant literature. Although we did not focus on this main effect, which is arguably of a lower theoretical importance than the robust cost asymmetry found in this study, its actual relationship to internal shielding remains to be established and explored in future studies. That being said, among the three competing accounts considered in this study, associative interference appears to provide the best one to account for the cost asymmetry observed here, which implies an imbalance in shielding between external and internal attention. 

external and internal attention. That is, an important potential implication of our results is that (internal) attention to memory can be shielded more efficiently than attention to perception.
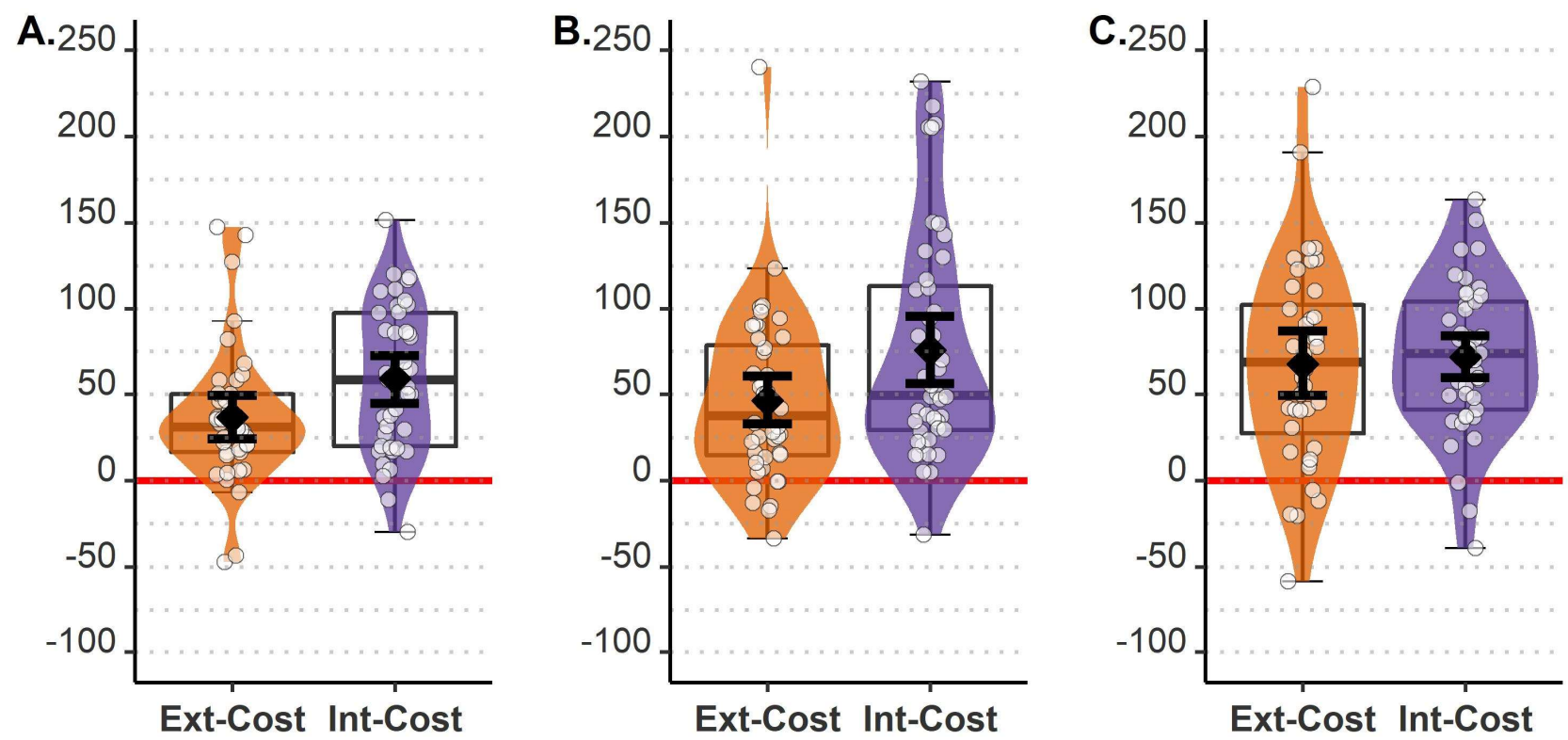

6

Figure 5. Switch costs for Experiment 1-3. In black: Mean response time and 95\% confidence interval (CI). In white: individual participants' means. A. Asymmetric switch cost in Experiment 1. B. Asymmetric in Experiment 2. C. Non-asymmetric cost in Experiment 3.

\section{More efficient shielding for internal than external attention?}

At face value, this imbalance in shielding implies that internal attention is more readily deployable - and more easily shielded - than external attention. This conclusion might seem counterintuitive given the historical bias for using external stimuli in attention research, but it can provide a useful new perspective on the relationship between external and internal attention. In addition, some indirect evidence for the idea of internal attention is dominant over external attention is available in the literature. 
First, a large body of neuroimaging data has shown that when participants are unconstrained by an external task, they reliably revert to a cognitive default mode ("resting-state"), characterized by increased activity in a network of midline and temporal brain regions (Raichle, 2015, for a review). This network has been associated with spontaneous, internally directed cognition, for example daydreaming, autobiographical memory retrieval or mentation about the future. This suggests that internally directed attention represents the 'default' cognitive mode when we are not actively engaged with an external task. Second, we know from mind-wandering research that, even when performing a task, attention often is drawn inwardly and 'mind-wandering' intrusions occur that disrupt task performance (Seli et al., 2016, for a review). Finally, research on WM guidance (i.e., internal attention) of external attention reports strong and automatic internal intrusions in external attention (e.g., Kiyonaga et al., 2012; Olivers et al., 2011). On the other hand, however, it has also been found that external distractors can disrupt internal maintenance in WM (e.g., Hakim et al., 2019; Whitehead et al., 2019). A direct and systematic comparison of these effects may prove insightful, but has to our knowledge not been carried out yet.

Alternatively, Tarder-Stoll and colleagues (2019) argue that the imbalance between external and internal attention is more state-dependent. The argument goes that the balance between external and internal attention is determined by 'encoding' and 'retrieval' states (see also Tulving, 2002). In the former state, attention is primarily externally oriented, whereas it is mostly internally oriented in the latter. Among other factors, exposure to novel stimuli promotes an (external) 'encoding' state by increasing acetylcholine levels in the hippocampus. Conversely, a familiar context reduces these levels and brings about a retrieval mode by reducing these levels. In the study presented here and in our previous work (Verschooren et al., 2019), we have re-used the same eight stimuli throughout the task, which may have potentially promoted a dominant retrieval mode.

Based on the new data presented here, it is not possible to distinguish between a more inherent versus more state-dependent view, but this issue represents an interesting avenue for further research. 


\section{Limitations}

While we observed strong evidence for the robustness of the asymmetric switch effect between internal and external attention, and also obtained strong evidence against a priming based explanation for that effect, it is important to emphasize that we did not find conclusive evidence in favor of associative interference over memory retrieval mediating that effect in the current series of studies. With this caveat in mind, it is however important to note that the Bayesian evidence in Experiment 3 goes in the direction of the absence of an effect, and the external switch cost did increase; two effects which are compatible solely with the associative interference account. Furthermore, in the literature, there is accumulating evidence in favor of associative interference and more broadly, memory representations that can modulate cognitive control (Abrahamse et al., 2016; Braem \& Egner, 2018; Egner, 2014; Mayr et al., 2014; Spape \& Hommel, 2008). Hence, it appears important to explore this question further in future studies, preferably using a variety of experimental procedures. In this context, it would probably be interesting to consider the possibility of an hybrid model, assuming that associative interference (procedural level) and memory retrieval (declarative level) might both contribute to the asymmetric switch cost found here. Finally, another important question raised by this work is whether the increased interference on external trials originates from the presence of competing external stimuli, from stimulus-location associations activated slower in general when performing an external single-task condition, which is somewhat unexpected. One potential explanation is that this condition is not very engaging in the absence of the competing internal 
1 interpretation of the asymmetric switch cost in terms of associative interference. At any rate, some caution

2 is needed in the interpretation of this effect, and further research is desirable to confirm that it is rooted

3 in associative interference, as we contend based on these results.

5

6

7 found when switching between (external) perception and (internal) memory. In comparison, priming and 8

\section{Conclusions}

We report evidence for the notion that associative interference can account for the cost asymmetry memory retrieval do not provide viable accounts of it. An important new hypothesis derived from this interpretation is that internal attention may be more easily shielded from external intrusions than the other way around. We discussed several potential factors creating this imbalance, but additional research is needed to establish which of them most likely causes it. More broadly, our findings provide further support for a currently developing framework that places high-level cognitive functions under learning and memory-driven control (Abrahamse et al., 2016; Braem \& Egner, 2018; Egner, 2014; Mayr et al., 2014; Spape \& Hommel, 2008). We advocate that this framework can also be used to explain attention flexibility, and more specifically the remarkable ability to switch between external and internal attention.
We thank Peter Whitehead for helpful comments on an earlier version of this draft. SV and GP are funded by a Concerted Research Action Grant from Ghent University (BOF16/GOA/017). This research was supported by a FWO grant for a long stay abroad (V402519N) awarded to SV. We declare no conflicts of interest. 
Abrahamse, E., Braem, S., Notebaert, W., \& Verguts, T. (2016). Grounding cognitive control in associative learning. Psychological Bulletin, 142(7), 693-728. https://doi.org/10.1037/bul0000047

Allport, D. A., Styles, E. A., \& Hsieh, S. (1994). Shifting intentional set: Exploring the dynamic control of tasks. Attention and Performance XV: Conscious and Nonconscious Information Processing., January 1994, 421-452. https://doi.org/10.1126/science.1134404

Barr, D. J., Levy, R., Scheepers, C., \& Tily, H. J. (2013). Random effects structure for confirmatory hypothesis testing: Keep it maximal. Journal of Memory and Language, 68(3). https://doi.org/10.1016/j.jml.2012.11.001

Braem, S., \& Egner, T. (2018). Getting a Grip on Cognitive Flexibility. Current Directions in Psychological Science, 27(6), 470-476. https://doi.org/10.1177/0963721418787475

Bryck, R. L., \& Mayr, U. (2008). Task selection cost asymmetry without task switching. Psychonomic Bulletin \& Review, 15(1), 128-134.

Bürkner, P.-C. (2017). Brms: An R Package for Bayesian Multilevel Models Using Stan. Journal of Statistical Software, 80(1). https://doi.org/10.18637/jss.v080.i01

Carlson, R. A., Wenger, J. L., \& Sullivan, M. A. (1993). Coordinating information from perception and working memory. Journal of Experimental Psychology: Human Perception and Performance, 19 3(3), 531-548. https://doi.org/10.1037/0096-1523.19.3.531

Chiu, Y.-C., \& Egner, T. (2017). Cueing cognitive flexibility: Item-specific learning of switch readiness. Journal of Experimental Psychology: Human Perception and Performance, 43(12), 1950-1960. https://doi.org/10.1037/xhp0000420

Chun, M. M., Golomb, J. D., \& Turk-Browne, N. B. (2011). A Taxonomy of External and Internal Attention. Annual Review of Psychology, 62(1), 73-101. https://doi.org/10.1146/annurev.psych.093008.100427

Dark, V. J. (1990). Switching between memory and perception: Moving attention or memory retrieval? Memory \& Cognition, 18(2), 119-127. https://doi.org/10.3758/BF03197088 
Dreisbach, G., \& Haider, H. (2008). That's what task sets are for: Shielding against irrelevant information. Psychological Research, 72(4), 355-361. https://doi.org/10.1007/s00426-007-0131-5

Dreisbach, G., \& Haider, H. (2009). How task representations guide attention: Further evidence for the shielding function of task sets. Journal of Experimental Psychology: Learning, Memory, and Cognition, 35(2), 477486. https://doi.org/10.1037/a0014647

Dreisbach, G., \& Wenke, D. (2011). The shielding function of task sets and its relaxation during task switching. Journal of Experimental Psychology: Learning, Memory, and Cognition, 37(6), 1540-1546. https://doi.org/10.1037/a0024077

Endo, N., Saiki, J., Nakao, Y., \& Saito, H. (2003). Perceptual judgments of novel contour shapes and hierarchical descriptions of geometrical properties. Shinrigaku Kenkyu : The Japanese Journal of Psychology, 74(4), $346-353$.

Frank, M. J., Loughry, B., \& Reilly, R. C. O. (2001). Interactions between frontal cortex and basal ganglia in working memory: A computational model. 1(2), 137-160.

Gilbert, S. J., \& Shallice, T. (2002). Task Switching: A PDP Model. Cognitive Psychology, 44(3), $297-337$. https://doi.org/10.1006/cogp.2001.0770

Hakim, N., Feldmann-Wüstefeld, T., Awh, E., \& Vogel, E. K. (2019). Perturbing Neural Representations of Working Memory with Task-irrelevant Interruption. Journal of Cognitive Neuroscience, 1-12. https://doi.org/10.1162/jocn_a_01481

Kass, R. E., \& Raftery, A. E. (1995). Bayes Factors. Journal of the American Statistical Association, 90(430), 773795. https://doi.org/10.1080/01621459.1995.10476572

Kiyonaga, A., Egner, T., \& Soto, D. (2012). Cognitive control over working memory biases of selection. Psychonomic Bulletin \& Review, 19(4), 639-646. https://doi.org/10.3758/s13423-012-0253-7

Kruschke, J. K. (2018). Rejecting or Accepting Parameter Values in Bayesian Estimation. Advances in Methods and Practices in Psychological Science, 1(2), 270-280. https://doi.org/10.1177/2515245918771304 
Lenth, R. (2018). Estimated Marginal Means, aka Least-Squares Means. https://doi.org/10.1080/00031305.1980.10483031

Lo, S., \& Andrews, S. (2015). To transform or not to transform: Using generalized linear mixed models to analyse reaction time data. Frontiers in Psychology, 6(August), 1-16. https://doi.org/10.3389/fpsyg.2015.01171

Logan, G. D. (1988). Toward an instance theory of automatization. Psychological Review, 95(4), $492-527$. https://doi.org/10.1037/0033-295X.95.4.492

Mayr, U., Kuhns, D., \& Hubbard, J. (2014). Long-term memory and the control of attentional control. Cognitive Psychology, 72, 1-26. https://doi.org/10.1016/j.cogpsych.2014.02.001

McElreath, R. (2016). Statistical rethinking: A Bayesian course with examples in $R$ and Stan.

Monsell, S. (2003). Task switching. Trends in Cognitive Sciences, 7(3), 134-140. https://doi.org/10.1016/S13646613(03)00028-7

Myers, N. E., Stokes, M. G., \& Nobre, A. C. (2017). Prioritizing Information during Working Memory: Beyond Sustained Internal Attention. Trends in Cognitive Sciences, 21(6), 449-461. https://doi.org/10.1016/j.tics.2017.03.010

Nalborczyk, L., Batailler, C., Vilain, A., \& Bürkner, P.-C. (2018). An Introduction to Bayesian Multilevel Models Using brms: A Case Study of Gender Effects on Vowel Variability in Standard Indonesian. 18.

Oberauer, K. (2002). Access to information in working memory: Exploring the focus of attention. Journal of Experimental Psychology: Learning, Memory, and Cognition, 28(3), 411-421. https://doi.org/10.1037/0278-7393.28.3.411

Oberauer, K. (2009). Chapter 2 Design for a Working Memory. In Psychology of Learning and Motivation (Vol. 51, pp. 45-100). Elsevier. https://doi.org/10.1016/S0079-7421(09)51002-X

Olivers, C. N. L., Peters, J., Houtkamp, R., \& Roelfsema, P. R. (2011). Different states in visual working memory: When it guides attention and when it does not. Trends in Cognitive Sciences, 15(7), 327-334. https://doi.org/10.1016/j.tics.2011.05.004 
Raichle, M. E. (2015). The Brain's Default Mode Network. Annual Review of Neuroscience, 38(1), $433-447$. https://doi.org/10.1146/annurev-neuro-071013-014030

Reilly, R. C. O., \& Frank, M. J. (2006). Making Working Memory Work: A Computational Model of Learning in the Prefrontal Cortex and Basal Ganglia. 328, 283-328.

Seli, P., Risko, E. F., Smilek, D., \& Schacter, D. L. (2016). Mind-Wandering With and Without Intention. Trends in Cognitive Sciences, 20(8), 605-617. https://doi.org/10.1016/j.tics.2016.05.010

Speelman, C. P., \& McGann, M. (2013). How Mean is the Mean? Frontiers in Psychology, 4, 451. https://doi.org/10.3389/fpsyg.2013.00451

Stroop, J. R. (1935). Studies of interference in serial verbal reactions. Journal of Experimental Psychology, 18(6), 643-662. https://doi.org/10.1037/h0054651

Tarder-Stoll, H., Jayakumar, M., Dimsdale-Zucker, H. R., Gunseli, E., \& Aly, M. (2019). Dynamic internal states shape memory retrieval [Preprint]. PsyArXiv. https://doi.org/10.31234/osf.io/kudj3

Tulving, E. (2002). Episodic Memory: From Mind to Brain. Annual Review of Psychology, 53(1), 1-25. https://doi.org/10.1146/annurev.psych.53.100901.135114

Vandierendonck, A., Liefooghe, B., \& Verbruggen, F. (2010). Task switching: Interplay of reconfiguration and

Vassena, E., Silvetti, M., Boehler, C. N., Achten, E., Fias, W., \& Verguts, T. (2014). Overlapping Neural Systems Represent Cognitive Effort and Reward Anticipation. PLoS ONE, 9(3), e91008. https://doi.org/10.1371/journal.pone.0091008

Verschooren, S., Liefooghe, B., Brass, M., \& Pourtois, G. (2019). Attentional Flexibility Is Imbalanced: Asymmetric Cost for Switches Between External and Internal Attention. Journal of Experimental Psychology: Human Perception and Performance, 45(10), 1399-1414.

Verschooren, S., Schindler, S., De Raedt, R., \& Pourtois, G. (2019). Switching attention from internal to external information processing: A review of the literature and empirical support of the resource sharing account. Psychonomic Bulletin \& Review, 26(2), 468-490. https://doi.org/10.3758/s13423-019-01568-y 
1 Wagenmakers, E.-J., Lodewyckx, T., Kuriyal, H., \& Grasman, R. (2010). Bayesian hypothesis testing for psychologists: A tutorial on the Savage-Dickey method. Cognitive Psychology, 60(3), 158-189. https://doi.org/10.1016/j.cogpsych.2009.12.001

Waszak, F., Hommel, B., \& Allport, A. (2003). Task-switching and long-term priming: Role of episodic stimulustask bindings in task-shift costs. In Cognitive Psychology. https://doi.org/10.1016/S0010-0285(02)005200

Whitehead, P. S., Ooi, M. M., Egner, T., \& Woldorff, M. G. (2019). Neural Dynamics of Cognitive Control over Working Memory Capture of Attention. Journal of Cognitive Neuroscience, 31(7), 1079-1090. https://doi.org/10.1162/jocn_a_01409

Wickham, H. (2016). Ggplot2: Create Elegant Data Visualisations Using the Grammar of Graphics.

Wickham, H. (2017). Tidyverse: Easily Install and Load the "Tidyverse" [R package tidyverse version 1.2.1].

Yeung, N., \& Monsell, S. (2003). Switching Between Tasks of Unequal Familiarity: The Role of Stimulus-Attribute and Response-Set Selection. Journal of Experimental Psychology: Human Perception and Performance, 29(2), 455-469. https://doi.org/10.1037/0096-1523.29.2.455 